\title{
Highly nondegenerate four-wave mixing and gain nonlinearity in a strained multiple-quantum-well optical amplifier
}

\author{
Jianhui Zhou, Namkyoo Park, Jay W. Dawson, and Kerry J. Vahala \\ Department of Applied Physics, Mail Stop 128-95, California Institute of Technology, Pasadena, \\ California 91125 \\ Michael A. Newkirk, Uziel Koren, and Barry I. Miller \\ AT\&T Bell Laboratories, Crawfords Corner Road, Holmdel, New Jersey 07733
}

(Received 19 November 1992; accepted for publication 10 March 1993)

\begin{abstract}
Highly nondegenerate four-wave mixing was investigated in a $1.5 \mu \mathrm{m}$ compressively strained multi-quantum-well semiconductor traveling-wave optical amplifier at detuning frequencies up to $600 \mathrm{GHz}$. A gain nonlinearity with a characteristic relaxation time of $650 \mathrm{fs}$ was determined from the data, and the nonlinear gain coefficient was estimated to be $4.3 \times 10^{-23} \mathrm{~m}^{3}$. Dynamic carrier heating is believed to be the major source of nonlinear gain in this device at the wavelengths investigated.
\end{abstract}

Nondegenerate four-wave mixing (NDFWM) in semiconductor gain media has been studied extensively over the past few years, ${ }^{1-4}$ because of both its role in improving understanding of nonlinear processes in semiconductors and its potential device applications. Two mechanisms have been proposed and demonstrated to be responsible for NDFWM in semiconductors. ${ }^{14}$. One is carrier density modulation caused by interband photomixing of pump and probe fields. This interband process generates gain and index gratings in the active layer, leading to significant NDFWM signals at frequencies below a few $\mathrm{GHz}$, corresponding to the spontaneous carrier lifetime $\tau_{S}$. Additional ultrafast gain saturation, referred to as the gain nonlinearity effect, is the other mechanism. In this case, the occupation probability of carriers in each energy band, rather than the actual carrier population, is modulated by the pump and probe waves. This intraband relaxation process is much weaker than the interband process at low frequencies, but, owing to its ultrafast dynamics, can dominate the NDFWM process at frequencies well beyond the corner frequency of the carrier density modulation.

Apart from its importance to NDFWM, the nonlinear gain effect has a significant impact on semiconductor laser modulation dynamics and spectral properties, especially for long wavelength devices. However, the physical mechanisms causing the gain nonlinearity are still not fully understood. Although certain proposed mechanisms ${ }^{5}$ would not contribute to NDFWM in traveling wave amplifiers (TWAs), two leading candidate mechanisms can cause a significant NDFWM effect. These are carrier heating and spectral hole burning. An early theoretical treatment of NDFWM in semiconductors by Agrawa ${ }^{1}$ considered spectral hole burning effects. Recent measurements of NDFWM by Kikuchi et al. ${ }^{2}$ employing a semiconductor TWA support this mechanism. However, femtosecond pumpprobe measurements of InGaAsP TWAs by Hall et al. ${ }^{6,7}$ earlier concluded that dynamic carrier heating was the major source of nonlinear gain. In addition, a NDFWM experiment by Tiemeijer ${ }^{3}$ supports the carrier heating theory. Further investigations are obviously needed to clarify this controversial issue.
In this experiment, three tunable erbium-doped fiber ring lasers ${ }^{8}$ were employed as pump, probe, and local oscillators to study NDFWM in a compressively strained quantum-well TWA operating at $1.5 \mu \mathrm{m}$. In addition to providing additional information on the nonlinear gain effect, this represents the first measurements by NDFWM of this mechanism in the strained quantum-well system. The TWA structure used in this study is described in Ref. 9, and the device length is $800 \mu \mathrm{m}$. The specific device used here was operated at a $150 \mathrm{~mA}$ bias current where it was measured to have a small signal gain of $16.3 \mathrm{~dB}$ for the TE mode, a $3 \mathrm{~dB}$ amplified spontaneous emission spectral width of $64 \mathrm{~nm}$, gain ripple $< \pm 0.1 \mathrm{~dB}$, and a saturation power of $5.66 \mathrm{~mW}$.

As shown in Fig. 1, light from the pump and probe lasers was combined using a 50/50 fiber coupler, and then coupled into the TWA after passing through a polarizer. The TWA gain was saturated to $14.5 \mathrm{~dB}$ by input power of $120 \mu \mathrm{W}$ from the erbium pump and probe lasers during the measurement. Two optical isolators providing $60 \mathrm{~dB}$ isolation were used to eliminate feedback caused by the facets of the input and output fibers. The NDFWM signal was heterodyne detected by mixing the signal with the fiber-laser local oscillator. The local oscillator was always tuned relative to the signal to provide a mixing frequency of $4 \mathrm{GHz}$, thereby ensuring that the electrical response of the detector

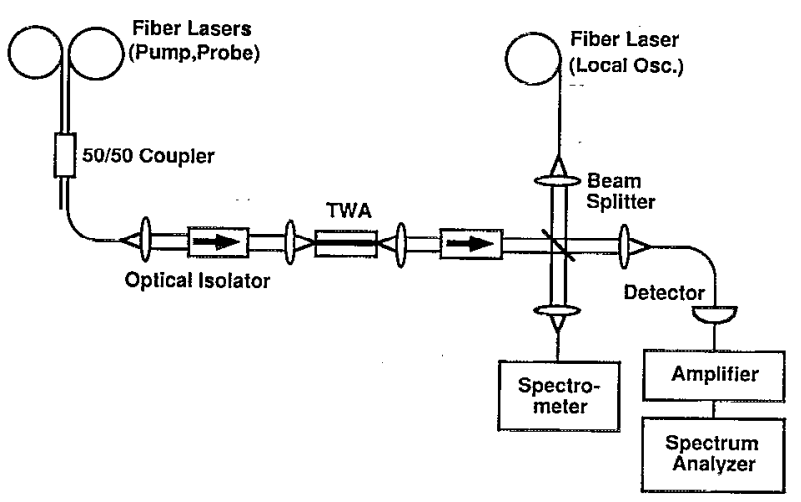

FIG. 1. Experimental setup for the NDFWM measurements. 


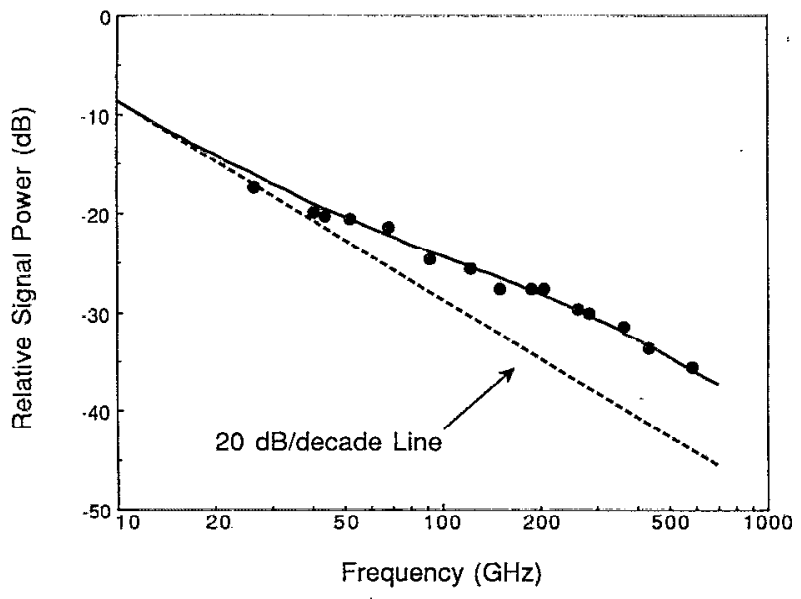

(a)

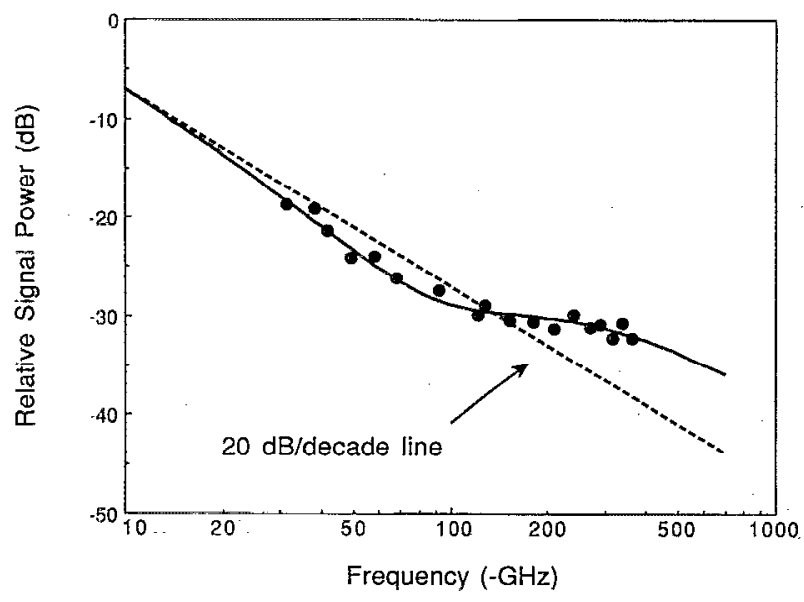

(b)

FIG. 2. Relative NDFWM signal power as a function of (a) positive detuning frequency, (b) negative detuning frequency.

and electronics would not affect the actual measured response. Detected photocurrent was amplified by a microwave amplifier and then measured using a spectrum analyzer. Because the fiber lasers used in this experiment have a quantum limited intensity noise and narrow linewidth $(<4 \mathrm{kHz}),{ }^{10}$ the system sensitivity was very high.

The NDFWM signal was measured for detuning frequencies from -400 to $600 \mathrm{GHz}$, limited only by the tuning range of one of the three fiber lasers used in this experiment. The relative signal power as a function of detuning frequency is plotted in Fig. 2. A corner frequency of about $200 \mathrm{GHz}$ is visible in the plot.

To further understand the measurement results, we modeled the process using the simplified formula introduced by Kikuchi et al.,

$$
E_{\mathrm{FWM}}=-\frac{1}{2} E_{p}^{2} E_{q}^{*}\left(\frac{1-i \alpha}{1-i 2 \pi f \tau_{s}} \frac{1}{I_{s}}+\frac{1-i \beta}{1-i 2 \pi f \tau_{n}} \frac{1}{I_{n}}\right),
$$

where $E_{\mathrm{FWM}}, E_{p}$, and $E_{q}$ are mode amplitudes of the signal, pump, and probe fields. $f$ is the detuning frequency defined as $f_{p}-f_{q}$, and $I_{s}$ and $\tau_{s}$ are the saturation power and the characteristic relaxation time governing the inter- band process, while $I_{n}$ and $\tau_{n}$ are the saturation power and the characteristic relaxation time associated with the nonlinear gain effect. In addition, $\alpha$ is the linewidth enhancement factor and $\beta$ is the ratio of real and imaginary parts of refractive index change induced by gain nonlinearity. $\beta$ has been calculated to be much less than unity for the case of spectral hole burning, ${ }^{1}$ but as high as 20 for the case of carrier heating in bulk layers. ${ }^{2}$ We performed a calculation for the case of a quantum-well system and find that depending on the operational wavelength, both positive and negative values with maximum magnitudes greater than unity are possible for $\beta$. In particular, negative values are obtained for the wavelengths used in the present measurement. Therefore, the $\beta$ parameter can serve, in some cases, as another criterion in determining the physical mechanism responsible for the nonlinear gain.

The relative signal power, after normalization, is given by

$$
I_{\mathrm{FWM}}=\left|\frac{1-i \alpha}{1-i 2 \pi f \tau_{s}} \frac{1}{I_{s}}+\frac{1-i \beta}{1-i 2 \pi f \tau_{n}} \frac{1}{I_{n}}\right|^{2} .
$$

As shown in Fig. 2, the above formula provides a good fit to the experimental data. The best fit gives $\tau_{n}=650 \mathrm{fs}$ and two other fitting parameters. This time constant of $650 \mathrm{fs}$ is in good agreement with previous measurements in both time and frequency domains of the dynamic carrier heating effect. ${ }^{3,6,7}$ Although other contributions to nonlinear gain with shorter characteristic time constants cannot be resolved in this experiment, our result clearly shows that dynamic carrier heating provides the major contribution to the gain nonlinearity in this measurement. In addition, the other two independent parameters used in the fit yield the following relations between $\alpha, \beta$, and the nonlinear gain coefficient $\epsilon$,

$$
\begin{aligned}
& \beta=-\frac{1.53 \alpha+1}{\alpha-1.53}, \\
& \epsilon=1.24 \frac{(\alpha-1.53) V_{g} A h v}{I_{s} \tau_{s}} .
\end{aligned}
$$

In Eq. (4) we have used the relation $\epsilon=V_{g} A h v / I_{n}$, where $V_{g}, A$, and $h v$ are the group velocity, mode size, and photon energy of the guided waves in the TWA. In addition, the units of $\tau_{s}$ in this expression are picoseconds.

To estimate the nonlinear gain coefficient $\epsilon$, we need to know the value of the $\alpha$ parameter. In gain spectra measurements ${ }^{11}$ on two DFB lasers having compressively strained multiple-quantum-well structures, it was determined to be 2.2 and 2.5 at the wavelength of $1.55 \mu \mathrm{m}$. However, the $\alpha$ parameter depends strongly on the injection carrier density, increasing rapidly when the density is high enough to saturate the occupancy of the lowest quantum-well subband. ${ }^{12,13}$ Since the carrier density is much higher in an amplifier than in a laser, where the density is clamped to the threshold value, we estimate a value of 6.5 for the $\alpha$ parameter in the TWA of this experiment. Using Eq. (3), we obtain $\beta=-2.2$, which is consistent with our calculation for the case of dynamic carrier heating effect (it would be nearly zero for the case of spec- 
tral hole burning). ${ }^{1}$ Parameters in Eq. (4) are chosen as follows: $\tau_{s}=200 \mathrm{ps}, V_{y}=7.5 \times 10^{7} \mathrm{~m} / \mathrm{s}, A=0.55 \mu \mathrm{m} \times 1.5$ $\mu \mathrm{m}, h \nu=0.807 \mathrm{eV}, I_{s}=5.66 \mathrm{~mW}$. The value for the nonlinear gain coefficient is then estimated to be $4.3 \times 10^{-23}$ $\mathrm{m}^{3}$, which is in close agreement with the range of values $5.93-9.73 \times 10^{-23} \mathrm{~m}^{3}$ determined in relative intensity noise measurements ${ }^{11,14}$ of lasers with compressively strained quantum-well active layers. However, in order to precisely determine the nonlinear gain coefficient, the exact values for the $\alpha$ parameter and the spontaneous lifetime $\tau_{s}$ under the experimental conditions must be known.

In view of the opposing conclusions previously reported $^{2,3,6,7}$ and the results of this experiment, we bclicve that both dynamic carrier heating and spectral hole burning contribute to the nonlinear gain, but that the strength of these two effects may vary due to differences in the active medium, operational wavelength, and other experimental conditions. TWA NDFWM measurement in the $\mathrm{THz}$ regime would be an ideal technique to study this issue since it cannot only resolve the time constant, but can also evaluate the relative contributions of both dynamic carrier heating and spectral hole burning to nonlinear gain in a single device under the same experimental conditions.

In summary, a NDFWM experiment in a compressively strained quantum-well TWA was performed using three fiber lasers as pump, probe, and local oscillators. The NDFWM signal was measured as a function of detuning frequency over the range $-400-600 \mathrm{GHz}$. The experimental data are in agreement with a calculation in which carrier heating is the primary cause of nonlinear gain. The nonlinear gain coefficient was estimated to be $4.3 \times 10^{-23}$ $\mathrm{m}^{3}$ and a carrier heating time constant of $650 \mathrm{fs}$ was mea- sured. In view of the two other NDFWM TWA measurements ${ }^{2,3}$ reported to date, it is concluded that both spcctral hole burning and dynamic carrier heating are important contributors to nonlinear gain. The conditions responsible for controlling the relative importance of these mechanisms are still under investigation.

This work was supported by the Office of Naval Research (Contract No. N00014-91-J-1524) and the Northrop Corp.

${ }^{1}$ G. P. Agrawal, J. Opt. Soc. Am. B 5, 147 (1988).

${ }^{2}$ K. Kikuchi, M. Kakui, C. E. Zah, and T. P. Lee, IEEE J. Quantum Electron. QE-28, 151 (1992).

${ }^{3}$ L. F. Tiemeijer, Appl. Phys. Lett. 59, 499 (1991).

${ }^{4}$ S. Murata, A. Tomita, J. Shimizu, M. Kitamura, and A. Suzuki, Appl. Phys. Lett. 58, 1458 (1991).

${ }^{5}$ C. B. Su, Electron. Lett. 24, 370 (1988).

${ }^{6}$ K. L. Hall, J. Mark, E. P. Ippen, and G. Eisenstein, Appl. Phys. Lett. 56, 1740 (1990).

${ }^{7}$ K. L. Hall, Y. Lai, E. P. Ippen, G. Eisenstein, and U. Koren, Appl. Phys. Lett. 57, 2888 (1990).

${ }^{8}$ N. K. Park, J. W. Dawson, K. J. Vahala, and C. Miller, Appl. Phys. Lett. 59, 2369 (1991).

${ }^{9}$ M. A. Newkirk, U. Koren, B. I. Miller, M. D. Chien, M. G. Young, T. L. Koch, G. Raybon, C. A. Burrus, B. Tell, and K. F. Brown-Goebeler, IEEE Photon Technol. Lett. 4, 1258 (1992).

${ }^{10}$ N. K. Park, J. W. Dawson, and K. J. Vahala, Opt. Lett. 17, 1274 (1992).

${ }^{11}$ H. Yasaka, K. Takahata, and M. Naganuma, IEEE J. Quantum Electron. QE-28, 1294 (1992).

${ }^{12}$ N. K. Dutta, H. Temkin, T. Tanbun-Ek, and R. Logan, Appl. Phys. Lett. 57, 1390 (1990).

${ }^{13}$ T. Ohtoshi and N. Chinone, IEEE Photon. Technol. Lett. 4, 117 (1992).

${ }^{14}$ T. Fukushima, J. E. Bowers, R. A. Logan, T. Tanbun-Ek, and H. Temkin, Appl. Phys. Lett. 58, 1244 (1991). 